\title{
O CONHECIMENTO DO CONTEXTO NA CONSTRUÇÃO DO CONHECIMENTO PEDAGÓGICO DO CONTEÚDO DOS FUTUROS PROFESSORES DE EDUCAÇÃO FÍSICA
}

\author{
Daniel Marcon \\ Universidade de Caxias do Sul, Caxias do Sul, Rio Grande do Sul, Brasil \\ Amândio Braga dos Santos Graça \\ Universidade do Porto, Porto, Portugal \\ Valmor Ramos \\ Universidade do Estado de Santa Catarina, Florianópolis, Santa Catarina, Brasil \\ Michel Milistetd \\ Universidade Estadual do Centro-Oeste, Guarapuava, Paraná, Brasil \\ Juarez Vieira Nascimento \\ Universidade Federal de Santa Catarina, Florianópolis, Santa Catarina, Brasil
}

\begin{abstract}
Resumo
Investiga-se como o conhecimento do contexto emerge nas práticas pedagógicas de estudantes-professores em diferentes etapas da formação. Quatro duplas de estudantes em Educação Física participaram da pesquisa qualitativa, cuja coleta de dados ocorreu por meio de entrevistas. Os resultados evidenciaram preocupações com a gestão da infraestrutura de aula, a logística e os recursos disponíveis. Conclui-se que as dificuldades encontradas no microcontexto impediram a contemplação de conhecimentos mais gerais na construção do conteúdo pedagógico. O programa de formação deve estimular a reflexão e a integração das três categorias de conhecimento ao longo de todo o curso.
\end{abstract}

Palavras-chave: Formação de professores. Ensino. Educação Física.

\section{Introdução}

O conjunto de conhecimentos necessários à atuação do professor, no sentido de alcançar os objetivos relacionados à sua formação profissional e devido à sua capacidade de intervenção pedagógica, é referido como a base do conhecimento para o ensino. (GRAÇA, 1997; GROSSMAN, 1990; SHULMAN, 1987).

Baseados nas formulações de Shulman (1987), verifica-se, nos referidos autores, que esse conjunto reúne conhecimentos acerca das características dos estudantes, dos conteúdos da matéria de ensino, da pedagogia geral, dos contextos de aprendizagem e da compreensão acerca do conhecimento pedagógico do conteúdo. Esse último exerce papel central, à medida que possibilita ao professor tornar o conteúdo compreensível aos estudantes.

Autores como Cochran, King e Deruiter (1991), O' Sullivan e Doutis (1994), Rovegno (2006, 2008), Rovegno e Dolly (2006), Schincariol (2002), Whipple (2002), entre outros, indicam a influência dos aspectos sociais, políticos, culturais e organizacionais do ambiente da sala de aula e na construção do conhecimento pedagógico do conteúdo. 
Estudos a respeito do conhecimento dos professores (GROSSMAN, 2008; GROSSMAN; MCDONALD, 2008; ROVEGNO, 2006; WHIPPLE, 2002) têm evidenciado a necessidade de os programas de formação inicial voltarem suas atenções à estrutura do conhecimento do contexto dos futuros docentes. Assim, é fundamental que o professor em formação obtenha uma compreensão aprofundada do contexto onde atuará, para adquirir condições de mobilizar os demais conhecimentos necessários à intervenção.

A atuação docente, de acordo com Shulman (1987), se dá a partir de três diferentes contextos: a) no trabalho com os estudantes; b) na administração e gestão escolar; c) em alcançar as particularidades sociais e culturais da comunidade onde a escola e os estudantes estão inseridos. Na presente investigação, essas três categorias do conhecimento do contexto foram denominadas, respectivamente, microcontexto da sala de aula, mesocontexto da escola e macrocontexto da comunidade. Todas são analisadas sob o ponto de vista de seus reflexos na qualificação pedagógica de futuros professores.

Além das importantes contribuições das propostas de Cochran, King e Deruiter (1991), Grossman (1990), Shulman (1987) e Zeichner (2010) para a compreensão do conhecimento do contexto, destacam-se também os estudos de Rink (1997) acerca do papel do contexto na aprendizagem docente, e também, a perspectiva ecológica de sala de aula de Siedentop (2002) para esclarecer a complexidade das relações que se estabelecem nas situações reais de aula.

O conhecimento do contexto adquire caráter específico e fundamental na formação dos futuros professores, à medida que emergem do contato direto com o ambiente de aula, dos papéis e das responsabilidades assumidas em todos os setores da escola. O presente estudo investiga como cada um dos três âmbitos do conhecimento do contexto: o micro, o meso e o macrocontexto emergem nas práticas pedagógicas de universitários em diferentes etapas da formação em Educação Física e como esse conhecimento participa na construção do conteúdo pedagógico dos futuros professores.

\section{Procedimentos metodológicos}

Para alcançar os objetivos da pesquisa, optou-se pela realização de um estudo de caso qualitativo com delineamento longitudinal misto (ALVES-MAZZOTTI; GEWANDSZNAJDER, 2001; DENZIN; LINCOLN, 2006; THOMAS; NELSON, 2002; YIN, 2010). A investigação foi aprovada por Comitê de Ética em Pesquisa com Seres Humanos (CEP/FUCS 286/2009). Os responsáveis pela instituição e cada um dos participantes assinaram o Termo de Consentimento Livre e Esclarecido de pesquisa.

\section{Voluntários da pesquisa}

A coleta de informações foi realizada com estudantes-professoras de um curso de Licenciatura em Educação Física com mais de 30 anos de existência. Os critérios utilizados para a seleção dos participantes foram: ser detentora da maior experiência acadêmica (melhores notas obtidas nas disciplinas já cursadas e maior número de créditos cursados); da maior experiência esportiva (maior tempo de prática esportiva competitiva e/ou participação em competições); da maior experiência pedagógica (maior tempo de atividade pedagógica autônoma ou como auxiliar em instituições de ensino fundamental, médio ou superior, clubes sociais, esportivos ou recreativos; empresas; hotéis). Participaram oito estudantes-professoras, com idade entre 18 e 22 anos, organizadas em quatro grupos que representavam as quatro etapas do curso de licenciatura, cada grupo composto por duas participantes. Integraram a pesquisa, especificamente, as estudantes de Educação Física com até $25 \%$ da carga horária do 
curso concluída (G1); entre 25 e $50 \%$ da carga concluída (G2); entre 50 e $75 \%$ da carga concluída (G3); e com mais de $75 \%$ da carga horária do curso concluída (G4).

\section{Instrumentos de coleta de dados}

A coleta de informações foi realizada durante três semestres letivos, período em que as estudantes-professoras avançaram em número de créditos cursados até seus respectivos e subsequentes grupos. A obtenção dos dados requereu uma entrevista inicial, um protocolo de autoavaliação da intervenção nas práticas pedagógicas e uma entrevista final. A entrevista inicial, semiestruturada, foi composta por questões abertas que, além de obterem informações sobre a biografia de cada estudante-professora, também questionavam sobre como cada uma interpretava a participação do conhecimento do contexto em suas práticas pedagógicas. Paralelamente à biografia, questionaram-se, também, o contexto de experiência de prática esportiva, o tempo de experiência, o tipo de prática, o nível da competição etc., e também, o tempo de experiência de prática pedagógica, o contexto da prática realizada e as características das estudantes. Para se obter os dados a respeito do conhecimento do contexto, os sujeitos foram questionados sobre: a) contexto escolar geral: a função e importância da escola, avaliações sobre as experiências obtidas na escola; b) contexto escolar específico: funções e importância das aulas e das práticas esportivas, avaliações pessoais dessas práticas; c) decisão de ser professora de Educação Física: motivos, importância, influências; d) expectativas quanto à formação inicial: características da instituição, expectativas e percepções da formação; e) planejamento de aula: como realiza seu plano, suas expectativas, quais os elementos, a importância; f) identificação e reflexão acerca das práticas pedagógicas proporcionadas na universidade para a construção do conhecimento.

As informações oriundas da entrevista inicial orientaram a estruturação do segundo instrumento na obtenção de informações sobre a intervenção das futuras professoras em diferentes modalidades de práticas pedagógicas. O protocolo de questões abertas e fechadas, relativas à autoavaliação de intervenção nas práticas pedagógicas, instigava a reflexão das estudantes-professoras acerca dos conhecimentos mais requisitados nas aulas ministradas. Assim, os temas indagados foram: a) fontes que contribuíram para a aquisição do conhecimento pedagógico; b) expectativas e reflexões a respeito da contribuição dos elementos do contexto na construção do conhecimento pedagógico.

A coleta de informações e o uso desse instrumento se estenderam pelos três semestres letivos da investigação, período que as estudantes-professoras preenchiam e devolviam, quinzenalmente, um protocolo de autoavaliação para cada uma das práticas pedagógicas ministradas na quinzena (ensino para os colegas, para os estudantes da comunidade nas IES ou nas escolas de Educação Básica, ou ainda, em suas práticas pedagógicas extracurriculares). As estudantes-professoras analisaram, ao longo do período da pesquisa, um total de 236 aulas (157 curriculares e 79 extracurriculares). O grupo G1 analisou 17 aulas curriculares e dois extracurriculares; o grupo G2, 30 aulas curriculares e 45 extracurriculares; o G3, 26 curriculares e 32 extracurriculares; e o G4, 84 aulas curriculares e nenhuma extracurricular.

A entrevista final, também semiestruturada, levou as estudantes-professoras a refletir sobre os resultados obtidos a partir da interpretação e análise da entrevista inicial e, fundamentalmente, sobre os protocolos de autoavaliação da intervenção nas práticas pedagógicas.

\section{Análise dos dados}

Após a transcrição e a aprovação das entrevistas inicial e final pelas participantes, bem como das respostas dissertativas do protocolo de autoavaliação, todas as informações foram 
tabuladas e categorizadas com o auxílio do software QSR NVivo, versão 8, e analisadas por meio da triangulação das diferentes fontes de informação entre si, ao lado do referencial teórico (ALVES-MAZZOTTI; GEWANDSZNAJDER, 2001; NEGRINE, 1999).

A adoção dos critérios de seleção dos participantes, dos instrumentos de coleta e dos procedimentos de análise de informações por meio de triangulação, teve como propósito estabelecer rigor científico a cada uma das etapas de realização do estudo, relativamente à preparação, à coleta de informações e à análise das inferências. Para efeito de apresentação e análise dos resultados, cada estudante-professora e seu respectivo grupo ou dupla são identificados no texto pelos seguintes pseudônimos: Aline e Amanda (G1); Bárbara e Bianca (G2); Camila e Carina (G3); Daiana e Dalila (G4).

\section{Resultados e Discussão}

As informações disponibilizadas pelas estudantes-professoras, por meio do protocolo de autoavaliação, foram apresentadas a partir das preocupações no âmbito do macrocontexto, do mesocontexto e do microcontexto, de acordo com a perspectiva ecológica de Siedentop (2002). As informações gerais relacionadas ao conhecimento do contexto, à distribuição da frequência e à taxa de referência por unidade de prática pedagógica dos quatro grupos, em cada uma das três categorias, são apresentadas na Tabela 1.

Tabela 1: Referência às categorias do conhecimento do contexto por unidade de prática, considerando as fases de realização do curso das estudantes-professoras.

\begin{tabular}{|c|c|c|c|c|c|}
\hline Grupos & $\begin{array}{c}\text { G1 } \\
\text { (Aline/ } \\
\text { Amanda) }\end{array}$ & $\begin{array}{c}\text { G2 } \\
\text { (Bárbara/ } \\
\text { Bianca) }\end{array}$ & $\begin{array}{c}\mathbf{G 3} \\
\text { (Camila/ } \\
\text { Carina) }\end{array}$ & $\begin{array}{c}\text { G4 } \\
\text { (Daiana/ } \\
\text { Dalila) }\end{array}$ & Total \\
\hline Práticas pedagógicas analisadas (PP) & 19 & 75 & 58 & 84 & 236 \\
\hline Conhecimento do contexto (CX) & 10 & 29 & 38 & 31 & 108 \\
\hline Racio CX/PP & 0,53 & 0,39 & 0,66 & 0,37 & 0,46 \\
\hline $\begin{array}{l}\text { Preocupações no âmbito do } \\
\text { macrocontexto }\end{array}$ & 0,00 & 0,00 & 0,00 & 0,00 & 0,00 \\
\hline $\begin{array}{l}\text { Preocupações no âmbito do } \\
\text { mesocontexto }\end{array}$ & 0,00 & 0,00 & 0,02 & 0,01 & 0,01 \\
\hline $\begin{array}{l}\text { Preocupações no âmbito do } \\
\text { microcontexto }\end{array}$ & 0,53 & 0,39 & 0,64 & 0,36 & 0,45 \\
\hline
\end{tabular}

Na Tabela 1 verifica-se que as preocupações com o microcontexto prevaleceram frente àquelas com o mesocontexto, enquanto que as apreensões com o macrocontexto não foram citadas nas reflexões. Quando se compara a evolução das preocupações com o microcontexto, verifica-se relativa oscilação de um grupo a outro, sendo os grupos G1 e G3 aqueles que despontam dos demais. No caso das preocupações com o mesocontexto, verificou-se disparidade nos resultados quanto ao microcontexto, seja nos grupos G1 e G2, à medida que nenhuma referência a esse âmbito foi citada, seja nos grupos G3 e G4, que mesmo o tendo referenciado, fizeram em frequência muito reduzida diante do microcontexto.

A exemplo do que foi observado no microcontexto, a taxa de referência por unidade de prática pedagógica, no racio CX/PP da Tabela 1, também destaca os grupos G1 e G3 diante dos grupos G2 e G4. O que se verifica, portanto, é que as estudantes-professoras dos quatro grupos pesquisados reconhecem a importância e a necessidade de se apropriarem da realidade do contexto onde atuarão. Porém, a atenção delas, manifestada em suas reflexões acerca das práticas pedagógicas, voltou-se, prioritariamente, ao microcontexto. 
Durante suas práticas, as integrantes do grupo G3 foram as que mais referenciaram o microcontexto. Como a estudante-professora Camila necessitava dedicar atenção especial para a solução dos problemas de indisciplina e de mau comportamento de seus estudantes, suas reflexões, frequentemente, se direcionaram para a sala de aula e para a relação dela com os estudantes e destes entre si. Segundo ela:

alguns estudantes se [agridem verbalmente], se empurram e não obedecem muito as instruções da professora. Continuo tendo dificuldade em "controlar" alguns estudantes no que diz respeito a suas atitudes [...]. Alguns estudantes se negaram a fazer os exercícios com determinados colegas. Ao final, para que a aula continuasse, tive que separá-los como que eles queriam. (Reflexões sobre as práticas pedagógicas - Camila).

Assim, Camila parece não ter considerado o potencial implícito nas informações oriundas dos meso e macrocontextos para a gestão e solução da indisciplina e mau comportamento dos estudantes, uma vez que não foram identificadas referências nesse sentido em suas reflexões.

Na comparação com os outros três grupos, as integrantes do G4 foram as que menos referenciaram o conhecimento do microcontexto. Acredita-se que tenham adquirido condições de direcionar conscientemente o foco de sua atenção, desviando-o do contexto mais imediato (microcontexto), e se voltado para outras categorias de conhecimento, como a dos estudantes, de conteúdo e pedagógico geral.

No caso específico do grupo G1, entendem-se como naturais as preocupações das estudantes-professoras com o microcontexto da sala de aula e com os estudantes, mesmo que estes sejam representados, respectivamente, pelas instalações da própria IES e por seus colegas, onde realizaram suas práticas. Alguns dos relatos das integrantes do grupo G1 exemplificam tais questões: "Por ter muitos estudantes na piscina, alguns se chocaram, até os professores (estudantes-professores) da raia entrarem em um acordo quanto à movimentação [dos estudantes] dentro da água." (Reflexões sobre as práticas pedagógicas - Amanda). "Os colegas estavam com frio e demoraram um pouco para participar ativamente da aula." (Reflexões sobre as práticas pedagógicas - Aline).

Como estão se iniciando na docência, é concebível que o foco principal de seu fazer pedagógico volte-se para o contexto mais próximo delas e que se sobressaia no cenário da prática pedagógica, devido ao potencial de influência imediata em sua autopercepção de competência docente. As constatações apresentadas evidenciam que as maiores preocupações das estudantes-professoras, independente do período do programa de formação inicial, não se referem às relações e influências da escola e da comunidade na aprendizagem dos estudantes. Pelo contrário, as principais preocupações dizem respeito, essencialmente, às implicações diretas de sua intervenção docente com os estudantes e ao alcance dos objetivos estabelecidos à sua prática pedagógica.

Destaca-se que a prioridade referida ao microcontexto pelas estudantes-professoras esteve ligada às condições de recursos disponíveis para a realização das práticas pedagógicas. Uma evidência disso é que suas reflexões remetiam, invariavelmente, ao espaço físico e à infraestrutura, além da quantidade e da qualidade dos recursos materiais a elas disponibilizados para as aulas: "A aula foi boa pelo local [...], pela quadra e materiais adequados." (Reflexões sobre as práticas pedagógicas - Bárbara). "Na aula de hoje o barulho da fábrica e de caminhões passando interferiu um pouco, pois tinha que falar muito alto e às vezes não me ouviam direito." (Reflexões sobre as práticas pedagógicas - Bianca).

Algumas vezes, as estudantes-professoras mencionaram a influência de observadores externos, isto é, de como professores e estudantes de outras turmas exerciam influência sobre a motivação dos alunos para se envolverem nas aulas e para falar sobre suas expectativas 
acerca do bom andamento das situações de aprendizagem. Essas questões podem ser observadas nos seguintes trechos: "A aula foi boa pela motivação apresentada pelas alunas quando há torcida ou pessoas assistindo." (Reflexões sobre as práticas pedagógicas - Aline). "Alguns estudantes ficaram prestando mais atenção no outro grupo do que na nossa aula, apesar de ser o mesmo plano de aula e eles saberem disso." (Reflexões sobre as práticas pedagógicas - Camila).

Outros elementos encontrados em suas reflexões, e que podem sugerir algum vínculo com o microcontexto, dizem respeito aos fatores climáticos e sua influência para que a prática pedagógica se realize conforme o planejado: "A aula de hoje foi melhor que as anteriores porque, além do tempo bom (lembrando: trabalho ao ar livre), as alunas estavam bem dispostas." (Reflexões sobre as práticas pedagógicas - Aline). "Havia muito barulho por causa da chuva, e eu não conseguia explicar." (Reflexões sobre as práticas pedagógicas Dalila).

Identificou-se, ainda, que as estudantes-professoras buscam obter informações sobre os estudantes acerca de seus conhecimentos, concepções e anseios, mas sem o propósito de contemplá-las em suas práticas pedagógicas. Para elas, parece que o microcontexto se refere antes aos fatores climáticos, estruturais e logísticos das situações de ensino e aprendizagem, ligados à infraestrutura, ao espaço físico e aos recursos materiais disponíveis, e depois, à gestão das relações dos estudantes com os conteúdos, com as atividades de ensino, com os interesses e realidade de vida dos estudantes, além do modo como esses fatores influenciam as aprendizagens, tal como propõem Grossman (2008), Whipple (2002), Rovegno e Dolly (2006), Rovegno (2008) e Schincariol (2002).

De modo geral, e conforme apontado por Mondéjar e Álvarez (2005), as estudantesprofessoras investigadas parecem ter problemas mais urgentes para resolver quando ministram suas práticas pedagógicas e seus estágios curriculares. Um exemplo disso é o aumento do nível de autopercepção de competência pela conquista da autonomia, da confiança, do respeito e do reconhecimento dos estudantes. Apesar de implícitas, essas preocupações foram recorrentes nas reflexões dos quatro grupos, o que pode ter dificultado o seu olhar para fora do microcontexto, nomeadamente para o mesocontexto da escola e para o macrocontexto da comunidade.

Essa constatação permite inferir que conhecimentos acerca do meso e macrocontextos participaram superficialmente da rotina acadêmica das estudantes-professoras, independentemente da modalidade das práticas pedagógicas que ministravam ou das etapas do programa de formação inicial em Educação Física que cursavam.

A inserção do conhecimento do contexto nos programas de formação inicial de professores desperta a atenção para a necessidade de se analisar como os cursos contemplam o ensino da Educação Física para estudantes em diferentes idades, como promovem a motivação e a participação dos estudantes em programas de atividades físicas, esportivas e de lazer, dentro e fora da escola, e o que os estudantes avaliam como significativo e estimulante (ROVEGNO, 2006, 2008; ABELL et al., 2009).

Em sua crítica sobre a falta de objetividade da literatura na abordagem dessas questões contextuais, além das bases que sustentam os programas de formação de professores, Grossman e McDonald (2008, p. 194, tradução nossa ) explicam que:

embora não sejam recentes os argumentos de que os programas de formação de professores necessitam ser concebidos de maneira contextualizada, são escassas as investigações que analisam a formação inicial sob esse ponto de vista. No campo da formação de professores, são relativamente poucas as pesquisas que têm investigado a maneira pela qual os contextos mais amplos, relativos às políticas educacionais, às características das escolas de Educação Básica e às demandas do mercado de trabalho se inter-relacionam e se inserem nos contextos específicos das IES para 
arquitetar os projetos de curso dos programas de formação de professores. A restrita consideração de tais fatores contextuais tornou difícil, tanto para os programas de formação reunir seus elementos estruturantes, quanto para os educadores e legisladores extrapolar questões relativas unicamente às demandas locais.

Mesmo reconhecendo alguns indícios de avanço nesse sentido, Ball e Forzani (2009) também advertem para a necessidade de se conhecer, pormenorizadamente, como essas relações acontecem e como incidem na formação de futuros professores na atuação docente e na aprendizagem de estudantes da Educação Básica. De fato, parece que a evolução do conhecimento do contexto de futuros professores somente ocorrerá quando puderem explorar e interagir diretamente com o contexto real de intervenção, por um período prolongado em tarefas que envolvam o microcontexto, o mesocontexto e o macrocontexto.

Dada a dificuldade de montar esse cenário e de alcançar todos esses objetivos dentro do período de realização do curso, é possível inferir que a verdadeira evolução do conhecimento do contexto não se dá no interior dos programas de formação inicial em Educação Física, mas após a conclusão desse estágio, ao se inserirem como professores em escolas de Educação Básica.

Essas ilações não os eximem da responsabilidade de planejar e de implementar, ao longo de todo o curso e não somente nos estágios curriculares, diferentes modalidades de práticas pedagógicas, conforme destacam autores como Behets e Vergauwen (2006), Grossman (2008), Metzler, Tjeerdsma e Mozen (2000), Rovegno (2006, 2008), Schincariol (2002), Grossman e McDonald (2008), Jenkins e Veal (2002), McCaughtry e Rovegno (2003), Rovegno (2006), Wiegand, Bulger e Mohr (2004 e Zeichner (2010).

Nesse sentido, os programas de formação inicial em Educação Física necessitam reconhecer a importância do conhecimento do contexto no processo de construção do conteúdo pedagógico de futuros professores, abordá-lo em diferentes disciplinas, sob vários ângulos e por meio de distintas estratégias. Com isso, poderá trazer os estudantes em idade escolar para a IES e, fundamentalmente, levar os professores em formação para dentro de escolas de Educação Básica desde o início do curso.

\section{Considerações finais}

As evidências encontradas indicam que as estudantes-professoras, independentemente do período do curso que representem, parecem ignorar o contexto escolar e as informações de vida dos estudantes, justamente pela grande atenção dedicada ao microcontexto onde se desenvolvem suas práticas docentes, isto é, na sua relação direta com os estudantes e nos resultados imediatos de suas intervenções pedagógicas.

Essa investigação não identificou participação incisiva do conhecimento do contexto, nos âmbitos meso e macro e no processo de construção do conhecimento pedagógico do conteúdo das participantes ao longo da formação inicial. Exceções à regra foram os momentos que antecederam a realização das práticas pedagógicas e dos estágios curriculares, quando conhecer o contexto se tornou uma prioridade para as estudantes-professoras e também para os professores-formadores. Nesses dois casos, identificaram-se alguns indícios de preocupação com o meso e macrocontextos, a saber, no planejamento, na implantação e na gestão das situações de ensino e aprendizagem.

As estudantes-professoras apresentaram dificuldades para alcançar a transposição de preocupações do microssistema para contextos mais amplos. Sendo assim, mesmo que a construção de conhecimentos por meio de experiências de aprendizagem possibilite demonstrar determinado conhecimento em um contexto específico, isso não significa que possam ser demonstrados em outros mesocontextos e macrocontextos. 
Os futuros professores somente poderão refletir sobre seus conhecimentos do contexto a partir de estratégias diversificadas que possam lhes oferecer possibilidades para se aproximar, conhecer, analisar, questionar e interagir com contextos educacionais e de vida dos estudantes. Acima de tudo, é necessário que, ao longo de toda a formação inicial, sejam estabelecidos mecanismos para estimular a reflexão e a percepção apurada da influência dos meso e macrocontextos no planejamento, na gestão das práticas pedagógicas, na interação com os estudantes e, principalmente, na aprendizagem e na formação dos próprios estudantes.

\title{
THE KNOWLEDGE OF THE CONTEXT IN CONSTRUCTION OF THE CONTENT'S EDUCATIONAL UNDERSTANDING OF FUTURE TEACHERS OF PHYSICAL EDUCATION
}

\begin{abstract}
The aim was to investigate how the knowledge of the context emerges in pedagogical practices of student teachers in different stages of formation. Four pairs of students in Physical Education took part in the qualitative research. The data were collected through interviews. The results showed concerns with the management of class infrastructure, logistics and available resources. The conclusion is that the difficulties encountered in microsystem prevented the contemplation of more general knowledge in the construction of pedagogical content. The training program must stimulate reflection and integration of the three categories of knowledge throughout the course.
\end{abstract}

Keywords: Teachers' development. Teaching. Physical Education.

\section{EL CONOCIMIENTO DEL CONTEXTO EN LA CONSTRUCCIÓN DEL CONOCIMIENTO PEDAGÓGICO DEL CONTENIDO DE FUTUROS PROFESORES DE EDUCACIÓN FÍSICA}

\section{Resumen}

Se investiga cómo el conocimiento del contexto emerge en las prácticas pedagógicas de estudiantes-profesores en las diferentes etapas de formación. Cuatro pares de estudiantes de Educación Física participaron en la investigación cualitativa y los datos fueron recolectados a través de entrevistas. Los resultados mostraron preocupaciones con la gestión de las clases y su infraestructura, la logística y los recursos disponibles. Se concluyó que las dificultades encontradas en el micro-contexto imposibilitaron que conocimientos más generales se incluyan en la construcción del contenido pedagógico. El programa de entrenamiento debe estimular la reflexión y la integración de las tres categorías de conocimientos a lo largo del curso.

Palabras-clave: Formación del profesorado. Enseñanza. Educación Física.

\section{Referências}

ABELL, S. K.; ROGERS, M. A. P.; HANUSCIN, D. L.; LEE, M. H.; GAGNON, M. J. Preparing the next generation of science teacher educators: a model for developing PCK for teaching science teachers. Journal of Science Teacher Education, v. 20, n. 1, p. 77-93, 2009.

ALVES-MAZZOTTI, A.; GEWANDSZNAJDER, F. O método nas ciências naturais e sociais: pesquisa quantitativa e qualitativa. São Paulo: Pioneira Thomson Learning, 2001. 
BALL, D. L.; FORZANI, F. M. The work of teaching and the challenge for teacher education. Journal of Teacher Education, v. 60, n. 5, p. 497-512, 2009.

BEHETS, D.; VERGAUWEN, L. Learning to teach in the field. In: KIRK, D.; MACDONALD, D.; O' SULliVAN, M. (Org.). Handbook of Physical Education. London: Sage, 2006, p. 407-424.

COCHRAN, K. F.; KING, R. A.; DERUITER, J. A. Pedagogical content knowledge: a tentative model for teacher preparation. In: ANNUAL MEETING OF THE AMERICAN EDUCATIONAL RESEARCH ASSOCIATION, 1991, Chicago. Anais... Chicago: American Educational Research Association, 1991.

DENZIN, N. K.; LINCOLN, Y. S. O planejamento da pesquisa qualitativa: teorias e abordagens. 2. ed. Porto Alegre: Artmed, 2006.

GRAÇA, A. O conhecimento pedagógico do conteúdo no ensino do basquetebol. 1997. Tese (Doutorado em Ciências do Desporto) - Faculdade de Desporto, Universidade do Porto, Porto, 1997.

GROSSMAN, P. The making of a teacher: teacher knowledge and teacher education. New York: Teachers College Press, 1990.

GROSSMAN, P. Responding to our critics: from crisis to opportunity in research on teacher education. Journal of Teacher Education, v. 59, n. 1, p. 10-24, 2008.

GROSSMAN, P.; MCDONALD, M. Back to the future: Directions for research in teaching and teacher education. American Educational Research Journal, v. 45, n. 1, p. 184-205, 2008.

JENKINS, J. M.; VEAL, M. L. Preservice teachers' PCK development during peer coaching. Journal of Teaching in Physical Education, v. 22, n. 1, p. 49-68, Oct., 2002.

MCCAUGHTRY, N.; ROVEGNO, I. Development of pedagogical content knowledge: Moving from blaming students to predicting skillfulness, recognizing motor development, and understanding emotion. Journal of Teaching in Physical Education, v. 22, n. 4, p. 355-368, July 2003.

METZLER, M. W.; TJEERDSMA, B. L.; MOZEN, D. M. Assessing pedagogical knowledge. Journal of Teaching in Physical Education, v. 19, n. 4, p. 487-507, jul. 2000.

MONDÉJAR, L.; ÁLVAREZ, F. Las preocupaciones docentes de los profesores noveles durante su período de prácticas didácticas. In: MONDÉJAR, L.; ÁLVAREZ, F. (Org.). La enseñanza de la Educación Física: análisis de las primeras experiencias docentes. Madrid: Editorial Síntesis, 2005, p. 143-160.

NEGRINE, A. S. Instrumentos de coleta de informações na pesquisa qualitativa. In: MOLINA NETO, V.; TRIVIÑOS, A. N. S. (Org.). A pesquisa qualitativa na Educação Física: alternativas metodológicas. Porto Alegre: Universidade/UFRGS/Sulina, 1999. p. 6194. 
O' SULLIVAN, M.; DOUTIS, P. Research on expertise: Guideposts for expertise and teacher education in Physical Education. Quest, v. 46, n. 2, p. 176-185, maio 1994.

RINK, J. E. Teacher education programs: the role of context in learning how to teach. JOPERD - The Journal of Physical Education, Recreation \& Dance, v. 68, n. 1, p. 17-24, jan. 1997.

ROVEGNO, I. Situated perspectives on learning. In: KIRK, D.; MACDONALD, D.; O'SUllivan, M. (Org.). The Handbook of Physical Education. London: Sage, 2006, p. 262-274.

ROVEGNO, I. Learning and instruction in social, cultural environments: Promising research agendas. Quest, v. 60, n. 1, p. 84-104, 2008.

ROVEGNO, I.; DOLLY, J. P. Constructivist perspectives on learning. In: KIRK, D.; MACDONALD, D.; O'SUllivan, M. (Org.). The Handbook of Physical Education. London. Sage, 2006, p. 242-261.

SCHINCARIOL, L. M. The types, sources, and perceived relevance of knowledge acquisition, and the enacted effects when teaching unfamiliar and familiar physical education content. Tese (Doutorado em Filosofia) - Department of Philosophy, The Ohio State University, Columbus, 2002.

SHULMAN, L. S. Knowledge and teaching: foundations of the new reform. Harvard Educational Review, v. 57, n. 1, p. 1-27, feb. 1987.

SIEDENTOP, D. Ecological perspectives in teaching research. Journal of Teaching in Physical Education, v. 21, n. 4, p. 427-440, jul. 2002.

THOMAS, J. R.; NELSON, J. K. Métodos de pesquisa em atividade física. 3. ed. Porto Alegre: Artmed, 2002.

WHIPPLE, C. E. Preservice teachers' views of content and pedagogical knowledge presented in the elementary component of a physical education teacher education program. Tese (Doutorado em Filosofia) - Department of Philosophy, The Ohio State University, Columbus, 2002.

WIEGAND, R. L.; BULGER, S. M.; MOHR, D. J. Curricular issues in Physical Education teacher education. JOPERD - The Journal of Physical Education, Recreation \& Dance, v. 75 , n. 8, p. 47-56, 2004.

YIN, R. K. Estudo de caso: planejamento e métodos. 4. ed. Porto Alegre: Bookman, 2010.

ZEICHNER, K. M. Rethinking the connections between campus courses and field experiences in college-and university-based teacher education. Journal of Teacher Education, v. 61, n. 1-2, p. 89-100, 2010. 
Recebido em: 22/03/2015

Revisado em: 22/12/2015

Aprovado em: 02/05/2016

Endereço para correspondência:

valmor.ramos@udesc.br

Valmor Ramos

Universidade do Estado de Santa Catarina, Centro de Educação Física e Desportos.

Rua Pascoal Simone, 358

Coqueiros

88080-350 - Florianópolis, SC - Brasil 